Check for updates

Cite this: Chem. Sci., 2018, 9, 6523

๑ All publication charges for this article have been paid for by the Royal Society of Chemistry

Received 30th April 2018

Accepted 3rd July 2018

DOI: $10.1039 / \mathrm{c} 8 \mathrm{sc} 01956 \mathrm{~h}$

rsc.li/chemical-science

\section{Identifying site-dependent reactivity in oxidation reactions on single Pt particles $\uparrow$}

\author{
Shahar Dery, ${ }^{\text {ab }}$ Suhong Kim, (D) ${ }^{c}$ David Haddad, ${ }^{\text {ab }}$ Albano Cossaro, (D) d \\ Alberto Verdini, (D) ${ }^{d}$ Luca Floreano, (D) ${ }^{d}$ F. Dean Toste (D) ${ }^{* c}$ and Elad Gross (D) *ab
}

Catalytic nanoparticles are heterogeneous in their nature and even within the simplest particle various surface sites exist and influence the catalytic reactivity. Thus, detailed chemical information at the nanoscale is essential for understanding how surface properties and reaction conditions direct the reactivity of different surface sites of catalytic nanoparticles. In this work, hydroxyl-functionalized $\mathrm{N}$ heterocyclic carbene molecules (NHCs) were anchored to the surface of Pt particles and utilized as chemical markers to detect reactivity variations between different surface sites under liquid and gas phase oxidizing conditions. Differences in the chemical reactivity of surface-anchored NHCs were identified using synchrotron-radiation-based infrared nanospectroscopy with a spatial resolution of 20 nanometers. By conducting IR nanospectroscopy measurements, along with complementary spatially averaged IR and X-ray spectroscopy measurements, we identified that enhanced reactivity occurred on the particles' periphery under both gas and liquid phase oxidizing conditions. Under gas phase reaction conditions the NHCs' hydroxyl functional groups underwent preferential oxidization to the acid along the perimeter of the particle. Exposure of the sample to harsher, liquid phase oxidizing conditions induced modification of the NHCs, which was mostly identified at the particle's periphery. Analysis of X-ray absorption spectroscopy measurements revealed that exposure of the sample to oxidizing conditions induced aromatization of the NHCs, presumably due to oxidative dehydrogenation reaction, along with reorientation of the NHCs from perpendicular to parallel to the Pt surface. These results, based on single particle measurements, demonstrate the high reactivity of surface sites that are located at the nanoparticle's periphery and the influence of reaction conditions on site-dependent reactivity.

\section{Introduction}

Molecular level understanding of catalytic processes is essential for the development of highly efficient and selective catalysts. ${ }^{\mathbf{1 4}}$ However, heterogeneities in the size, structure and composition of solid catalytic particles makes it difficult to directly monitor and identify the influence of various physicochemical parameters on the catalytic reactivity and selectivity. ${ }^{5-7}$ Thus, nondisruptive, detailed chemical information at the nanoscale is required for understanding how surface properties direct the reactivity of catalytic particles. ${ }^{8-10}$

Conventional spectroscopic techniques (such as infrared, ultraviolet-visible and Raman spectroscopy) for molecular

${ }^{a}$ Institute of Chemistry, The Hebrew University of Jerusalem, Jerusalem 91904, Israel. E-mail: elad.gross@mail.huji.ac.il

${ }^{b}$ The Center for Nanoscience and Nanotechnology, The Hebrew University of Jerusalem, Jerusalem 91904, Israel

${ }^{\circ}$ Department of Chemistry, University of California, Berkeley, California 94720, USA. E-mail:fdtoste@berkeley.edu

${ }^{d}$ CNR-IOM, Laboratorio Nazionale TASC, Basovizza SS-14, Trieste 34012, Italy

$\dagger$ Electronic supplementary information (ESI) available. See DOI: 10.1039/c8sc01956h analysis of catalytic reactions provide critical molecular level insight into the catalytic process, but are typically limited in their spatial resolution to the micrometer scale. ${ }^{11-15}$ Near-field microscopy approaches, such as tip-enhanced Raman spectroscopy (TERS) and infrared nanospectroscopy, can overcome such diffraction limitations by spatially confining light-matter interactions. ${ }^{\mathbf{1 6 - 2 6}}$ These two complementary apertureless approaches overcome the diffraction limit of light to detect the vibrational spectrum of molecules on surfaces with a spatial resolution of $\sim 20 \mathrm{~nm}$. TERS and IR nanospectroscopy measurements provide the technical capabilities to identify chemical processes at the nanoscale. However, the specific detection of reactants-into-products transformations on the surface of a single nanoparticle is not trivial even while using apertureless methods, since other competing molecular processes, including adsorption, diffusion and desorption, simultaneously occurs on the catalyst's surface..$^{5,11,27}$

High spatial resolution detection of chemical processes on surfaces can be achieved by using surface-anchored molecules as indicators for chemical reactivity. ${ }^{\mathbf{1 8 , 2 1 , 2 2}}$ For example, chemically active $\mathrm{NO}_{2}$ - and $\mathrm{NH}_{2}$-functionalized thiols were anchored to $\mathrm{Ag}$ and $\mathrm{Au}$ surfaces and chemical changes in the thiols 
functional groups, following exposure of the sample to various reaction conditions, were probed by TERS measurements. ${ }^{\mathbf{1 8 , 2 1}}$ We have recently demonstrated a different approach for high spatial resolution mapping of chemical reactions on catalytic particles. ${ }^{23}$ In this approach, N-heterocyclic carbene molecules (NHCs) with chemically-active functional groups were anchored to the surface of transition metal particles. The high stability, chemical versatility and ease of preparation of NHCs make them optimal chemical indicators for surface-induced reactivity. The surface-induced reactivity of NHCs was mapped using high spatial resolution synchrotron-based infrared nanospectroscopy (SINS) measurements. ${ }^{23}$ Using this approach, it was observed that the periphery of the particles, which contain higher density of metal atoms with low coordination numbers, in comparison to the flat regions on top of the particles, were more active in catalyzing oxidation and reduction of the chemically active groups in surface-anchored NHCs.

Recent works suggested that variations in the reaction conditions, for example the phase of the reaction, can result in changes to the mechanism and kinetics of oxidation reactions on Pt nanoparticles. ${ }^{28,29}$ Moreover, it is reasonable to posit that changes in the reaction conditions will differentially impact the various surface sites. However, support for this hypothesis is limited due to the paucity of nanoscale analysis of how modifications in reaction conditions influence the site-dependent reactivity of catalysts. ${ }^{30}$

Therefore, the aim of this study is to identify, by conducting high spatial resolution single-particle measurements, the ways by which exposure to liquid and gas phase oxidizing conditions modify the chemical reactivity of different surface sites. To achieve this goal we have used $\mathrm{OH}$-functionalized NHCs as indicators for surface-induced reactivity. The NHCs were anchored to the surface of Pt particles so that their functional groups reside in close proximity to catalytically active surface sites. As a consequence, the hydroxyl group can be oxidized upon exposure of the sample to oxidizing conditions (Fig. 1a).

High-spatial-resolution SINS measurements were conducted, following exposure of the sample to various oxidizing conditions, to identify site-dependent reactivity on single particles (Fig. 1b). Complementary, surface-sensitive infrared reflection absorption spectroscopy (IRRAS), X-ray photoelectron spectroscopy (XPS) and near-edge X-ray absorption fine structure (NEXAFS) measurements were performed to identify how exposure of the sample to gas- or liquid-phase oxidizing conditions impacted the chemical and structural properties of NHCs (Fig. 1c).

It should be noted that NHCs were initially developed as ligands in organometallic complexes. ${ }^{31}$ In the last decade it was widely demonstrated that these molecules can be efficiently anchored to metal surfaces. ${ }^{32-35}$ The structure and stability of surface anchored NHCs were studied by several surfacesensitive techniques. ${ }^{36-38}$ However, in depth analysis of the chemical and structural properties of chemically-active NHCs, which were recently utilized as indicators for surface induced reactivity, ${ }^{23,25}$ remains rare.

In this work, we integrated spatially localized (singleparticle) and averaged measurements to identify structure- reactivity correlation on single $\mathrm{Pt}$ particles under gas and liquid phase oxidizing conditions. In both cases, higher reactivity was detected on the particles' perimeter. Under gas phase reaction conditions the enhanced reactivity on the particle's periphery led to oxidation of the hydroxyl functional groups into acid. Exposure of the sample to harsher, liquid phase oxidizing conditions induced decarboxylation, which was followed by oxidation, in NHCs that were anchored to the particle's perimeter. The chemical changes in the NHCs' properties were coupled with changes in the NHCs' orientation from perpendicular to parallel to the Pt surface.

\section{Methods and instruments}

\section{Sample preparation}

Pt particles were prepared by evaporation of metallic Pt film on $\mathrm{SiO}_{2} / \mathrm{Si}(110)$ surface. This process was followed by annealing of the Pt-coated surface to $823 \mathrm{~K}$ for 5 hours, which was conducted under $\mathrm{N}_{2}$ environment to prevent coating of the metallic particles with a $\mathrm{SiO}_{2}$ layer. NHCs were prepared and activated in a glove box according to a recently published protocol. ${ }^{23}$ The freshly prepared carbene solution was transferred into a vial and covered the Pt-coated Si surface. After $48 \mathrm{~h}$, the Si wafer was removed from the glove box and washed three times with THF $(5 \mathrm{ml})$ and distilled water $(5 \mathrm{ml})$ intermittently. The sample was then flushed with $\mathrm{N}_{2}$ for $5 \mathrm{~min}$ and stored in a glove box.

\section{SINS measurements}

Synchrotron infrared light (provided by the Advanced Light Source, Lawrence Berkeley National Laboratory) was focused onto the apex of an oscillating Pt-Si AFM tip (Nanosensors, PtSi$\mathrm{NCH}$ ) at frequency $\omega$ in a modified commercial AFM (Innova, Bruker). Because the near-field scattered signal depends nonlinearly on the distance between the tip and the sample, the tip oscillation induces higher harmonics $(n \omega)$ in the near-field scattered signal. As a result, the near-field signal can be differentiated from the far-field background by detecting the high harmonic frequency $2 \omega$ with a lock-in amplifier. A modified commercial FTIR spectrometer (Nicolet 6700, Thermo Scientific) was used to collect the infrared nanospectroscopy signal. Following AFM topography imaging of the surface, infrared nanospectroscopy point measurements were conducted at selected locations of the particles' surface. The Fourier transform of the interferogram provides a complex-valued near-field spectrum. The real $(\operatorname{Re}(\nu)$, where $\nu$ is the wavenumber) and imaginary $(\operatorname{Im}(\nu))$ spectra can be represented as spectral amplitude $(A(\nu))$ and phase $(\varphi(\nu))$. The near-field spectra were reported in the form of a normalized scattering phase $(\varphi(\nu)=$ $\left.\varphi_{\text {sample }}(\nu)-\varphi_{\text {reference }}(\nu)\right)$, using the bare Si surface as a reference point.

SINS measurements were performed before and after exposure of the sample to liquid or gas phase oxidizing conditions. All SINS measurements were conducted at room temperature under nitrogen atmosphere. IR nanospectroscopy measurements were conducted on different sites on the surface of at least 10 particles under each reaction conditions. In these 


\section{a. NHCs on Pt NPs}

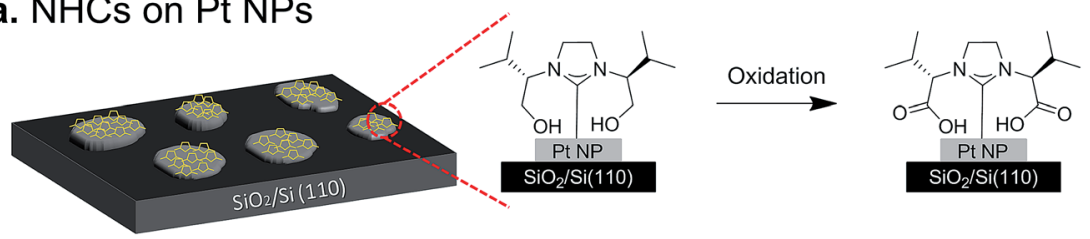

b. IR Nanospectroscopy

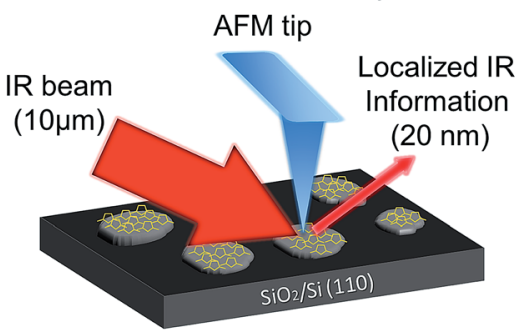

c. Ensemble-based IRRAS and NEXAFS

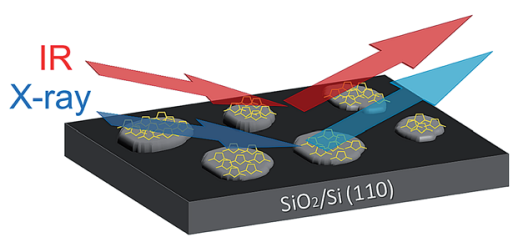

Fig. 1 Schematic representation of the experimental approach: (a) $\mathrm{OH}$-functionalized NHCs (marked with orange pentagons) were anchored to the surfaces of Pt particles (grey circles). The particles were supported on a $\mathrm{SiO}_{2} / \mathrm{Si}(110)$ wafer. Under oxidizing conditions the catalytically active Pt particles oxidize the hydroxyl group of the anchored NHCs. (b) High spatial resolution Synchrotron Infrared Nanospectroscopy (SINS) measurements were performed to identify site-dependent reactivity on single particles. In this setup, an AFM tip acts as an optical antenna by localizing the diffraction-limited IR beam (which has a focal spot of about $10 \mu \mathrm{m}$ ) and inducing an IR scattering signal with a tip-limited spot size of $\sim 20 \mathrm{~nm}$. (c) Chemical and structural characterization of surface-anchored NHCs was achieved by conducting ensemble-based Near Edge Xray Absorption Fine Structure (NEXAFS), X-ray photoelectron spectroscopy (XPS) and Infrared Reflection Absorption Spectroscopy (IRRAS) measurements.

measurements at least 8 out of 10 particles, on average, showed a similar trend in their site-dependent reactivity.

In all the performed IR nanospectroscopy measurements it was verified that the AFM tip is positioned either on the center or along the profile of the metallic particles. Therefore, the IR nanospectroscopy signal was mostly induced by molecules that were anchored on the various metal sites with only a minor, if any, contribution from molecules that reside on the metal/ metal-oxide interface.

\section{IRRAS measurements}

Measurements were conducted at room-temperature under vacuum on reflection-absorption cell (Harrick, Inc.) with FTIR spectrometer (Vertex V70, Bruker). Measurement parameters: 1024 scans with a resolution of $4 \mathrm{~cm}^{-1}$ using mercury cadmium telluride (MCT) detector.

\section{NEXAFS and XPS measurements}

X-ray photoelectron spectroscopy (XPS) and near-edge X-ray absorption experiments (NEXAFS) measurements were performed at the ALOISA beamline of the ELETTRA synchrotron facility in Trieste (Italy). ${ }^{39} \mathrm{X}$-ray absorption spectra measurements were taken in partial electron yield using a channeltron detector equipped with a front grid biased at negative voltage $(-230 \mathrm{~V})$ to filter out the low energy secondary electrons. The NEXAFS spectra at the carbon K-edge were measured with the resolution set to $\sim 80 \mathrm{meV}$, keeping the sample at a constant grazing angle of $6^{\circ}$. The orientation of the surface with respect to the photon beam polarization was changed from spolarization to close to p-polarization by rotating the sample coaxially to the photon beam axis. NEXAFS spectra were reported in the form of a normalized absorption amplitude $\left(I_{(\mathrm{E})}=\right.$

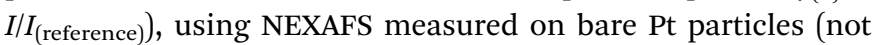
coated with NHCs) as a reference. X-ray photoelectron spectra of C1s, N1s and Pt4f were acquired. The binding energies of N1s $\mathrm{XP}$-spectra were calibrated according to the binding energy of the $\mathrm{C}-\mathrm{C}$ peak position $(285.5 \mathrm{eV})$ in the $\mathrm{C} 1 \mathrm{~s}$ spectra. Analysis of the XPS peaks and their fitting was performed using CasaXPS software.

\section{Results and discussion}

Pt particles with an average size of $100 \pm 30 \mathrm{~nm}$ were prepared by annealing $\left(823 \mathrm{~K}, 5 \mathrm{~h}, 1 \mathrm{~atm} \mathrm{~N}_{2}\right)$ of a $20 \mathrm{~nm}$ Pt film that was deposited on $\mathrm{SiO}_{2} / \mathrm{Si}(110)$ wafer (Fig. S1 $\dagger$ ). OH-functionalized NHCs were anchored on the supported Pt particles according to a recently published protocol. ${ }^{23}$ No detectable changes in the particles' morphology were observed following the attachment of NHCs on their surface (Fig. S1†).

IR nanospectroscopy measurements were performed to identify the NHCs' reactivity on Pt particles following exposure of the sample to gas-phase $\left(1 \mathrm{~atm} \mathrm{O}_{2}, 50{ }^{\circ} \mathrm{C}, 10 \mathrm{~h}\right)$ and liquidphase $\left(0.1 \% \mathrm{w} / \mathrm{w} \mathrm{H}_{2} \mathrm{O}_{2}, \mathrm{rt}, 10 \mathrm{~h}\right)$ oxidizing conditions. No additional chemical changes were obtained in the properties of surface-anchored NHCs while conducting longer-duration (beyond 10 hours) exposure time, ensuring that the surface anchored molecules reached their local chemical equilibrium.

AFM measurements mapped the topography of NHC-coated Pt particles (left panels, Fig. 2). Subsequently, single point SINS measurements were conducted at the center of the particles, yielding the corresponding IR spectra (right panels, Fig. 2). The 

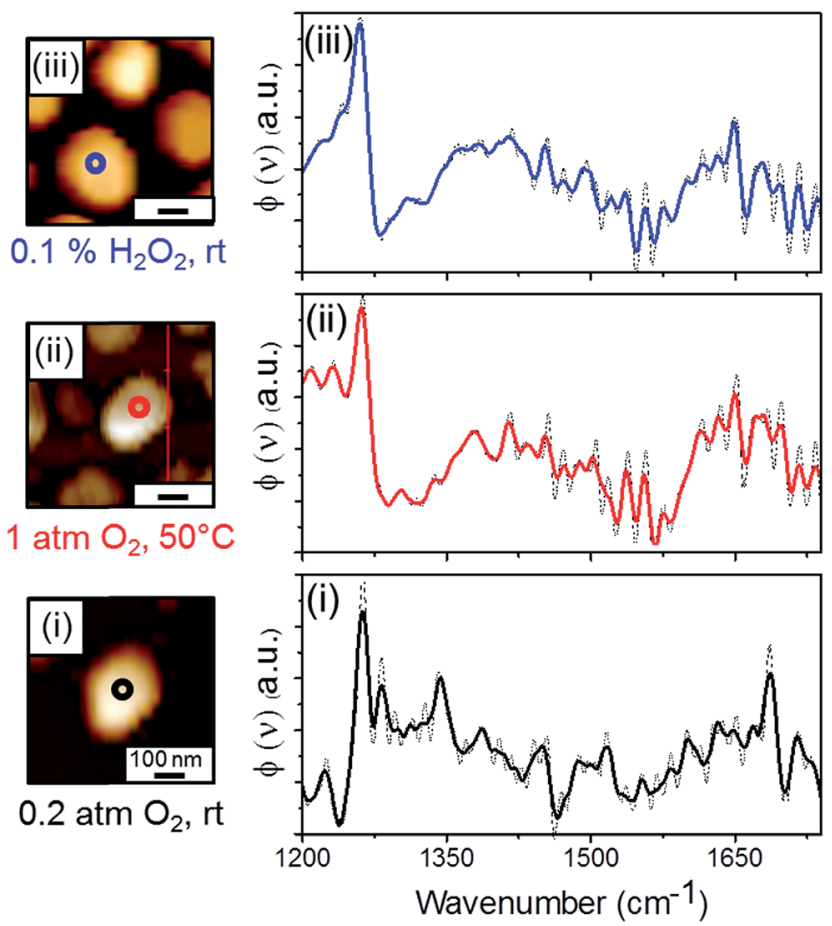

Fig. 2 AFM topography images (left panels) and single point SINS measurements (right panels) of Si-supported Pt particles that were coated with $\mathrm{OH}$-functionalized NHCs. IR nanospectroscopy measurements were conducted at the center of single Pt particles (the position of each IR measurement is marked by colored circles) after NHCs' deposition (i) and following exposure of the sample to gas phase ( $1 \mathrm{~atm} \mathrm{O}_{2}, 50{ }^{\circ} \mathrm{C}, 10 \mathrm{~h}$ ) (ii) and liquid phase $\left(0.1 \mathrm{w} / \mathrm{w} \% \mathrm{H}_{2} \mathrm{O}_{2}, \mathrm{rt}, 10\right.$ h) (iii) oxidizing conditions. The dash and color lines represent the asreceived and smoothed spectra, respectively.

location of each SINS measurement is marked by a colored circle in the AFM image.

SINS measurement was performed following exposure of the sample to air, with the AFM tip located above the center of the NHC-coated Pt particle (marked with a black circle in Fig. 2, AFM image (i)). An infrared spectrum with few vibrational peaks was obtained (Fig. 2, spectrum (i)). The main peak at $1250 \mathrm{~cm}^{-1}$ was assigned to $\mathrm{C}-\mathrm{OH}$ vibration. A minor peak was detected at $\sim 1700 \mathrm{~cm}^{-1}$ that can be correlated to $\mathrm{C}=\mathrm{O}$ stretch. However, its relatively small weight compared to the dominant $\mathrm{C}-\mathrm{OH}$ vibration indicates that the majority of the NHCs' hydroxyl groups were not oxidized after their exposure to air.

The infrared spectrum collected after exposure of the sample to gas phase oxidizing conditions (measurement position is marked with a red circle in Fig. 2, AFM image (ii)) exhibited a peak at $1250 \mathrm{~cm}^{-1}$ and a broad contribution at $1600-1700 \mathrm{~cm}^{-1}$, with integrated intensity larger than that region in spectrum (i) (Fig. 2, spectrum (ii)). The detection of an IR contribution at $\sim 1650 \mathrm{~cm}^{-1}$ and the fact that the intensity of the $\mathrm{C}-\mathrm{OH}$ vibration peak was unchanged after exposure of the sample to gas phase oxidizing conditions indicate that the $\mathrm{OH}$-functionalized molecules that reacted under these conditions were oxidized into acid, with no detectable formation of aldehyde. The higher noise level and the features at $1400-1600 \mathrm{~cm}^{-1}$ can be related to atmospheric water lines.

The NHC-coated Pt particles were also exposed to liquid phase oxidizing conditions, where $\mathrm{H}_{2} \mathrm{O}_{2}$ was used as an oxidizing agent $\left(0.1 \% \mathrm{w} / \mathrm{w} \mathrm{H}_{2} \mathrm{O}_{2}, \mathrm{rt}, 10 \mathrm{~h}\right)$. Single point SINS measurement was performed at the center of a single Pt particle (measurement position is marked with a blue circle in Fig. 2, AFM image (iii)). Two main peaks, at $1250 \mathrm{~cm}^{-1}$ and at $1600-$ $1700 \mathrm{~cm}^{-1}$, were obtained in the SINS spectrum and assigned to $\mathrm{C}-\mathrm{OH}$ and $\mathrm{C}=\mathrm{O}$ vibrations, respectively (Fig. 2, spectrum (iii)).

Interestingly, although $\mathrm{H}_{2} \mathrm{O}_{2}$ is a much more reactive oxidizing agent than $\mathrm{O}_{2}$, the acquired SINS spectrum was similar to the one obtained after exposure of the sample to gas phase oxidizing conditions. The high similarity of the two SINS spectra (Fig. 2, spectrum (ii) and (iii)) reveals that exposure of the sample to liquid phase oxidizing conditions did not noticeably change the NHCs' chemical properties or induced NHCs' deformation, in comparison to the results obtained following exposure of the sample to gas phase oxidizing conditions.

Exposure of the sample to reducing conditions $\left(1 \mathrm{~atm} \mathrm{H}_{2}\right.$, $80{ }^{\circ} \mathrm{C}, 10 \mathrm{~h}$ ), after its exposure to liquid or gas phase oxidizing conditions, led to a considerable decrease in the $\mathrm{C}=\mathrm{O}$ stretch regions, indicating that the oxidized functional groups of the NHCs can be reduced back to hydroxyl (Fig. S2 $\dagger$ ). ${ }^{23}$

One of the advantages in conducting high spatial resolution IR nanospectroscopy measurements is the ability to identify the influence of different surface sites on the reactivity. Herein, sitedependent reactivity was identified by comparing SINS spectra that were measured on the center and the periphery of a single Pt particle, following exposure of the sample to various oxidizing conditions. Differences in the reactivity on various surface sites were identified by subtracting the spectrum that was measured at the particle's perimeter $\left(\varphi_{(\nu) \text { side }}\right)$ from the one measured at the particle's center $\left(\varphi_{(v) \text { center }}\right)$ (Fig. 3). An example for the acquired SINS spectra at the side and center of the same particle is shown in Fig. S3. $\dagger$

SINS difference $\left(\varphi_{(v) \text { side }}-\varphi_{(v) \text { center }}\right)$ spectrum of NHC-coated Pt particles that were exposed to air did not show any noticeable signal (Fig. 3, spectrum (i)). Thus, almost no difference was detected between the chemical properties of NHCs that were anchored to the side and center of a Pt particle. This result indicates that under ambient conditions different surface sites do not induce detectable changes in the reactivity. Moreover, the similarity in the IR nanospectroscopy signals on various surface sites demonstrates that the local spectroscopic signal is not influenced by the position of the near-field measurement.

However, once the sample was exposed to gas phase oxidizing conditions $\left(1 \mathrm{~atm} \mathrm{O}_{2}, 50^{\circ} \mathrm{C}, 10 \mathrm{~h}\right)$, clear features were obtained in the difference spectrum (Fig. 3, spectrum (ii)). Two peaks at 1200 and $1700 \mathrm{~cm}^{-1}$ were detected in the difference spectrum and were correlated to $\mathrm{C}-\mathrm{OH}$ and $\mathrm{C}=\mathrm{O}$ vibrations. This result reveals that exposure of the sample to mild gas phase oxidizing conditions led to preferential oxidation of the hydroxyl functional groups in NHCs that were anchored on the particle's edge. It is hypothesized that the reason for the enhanced reactivity across the periphery of the particle is the 

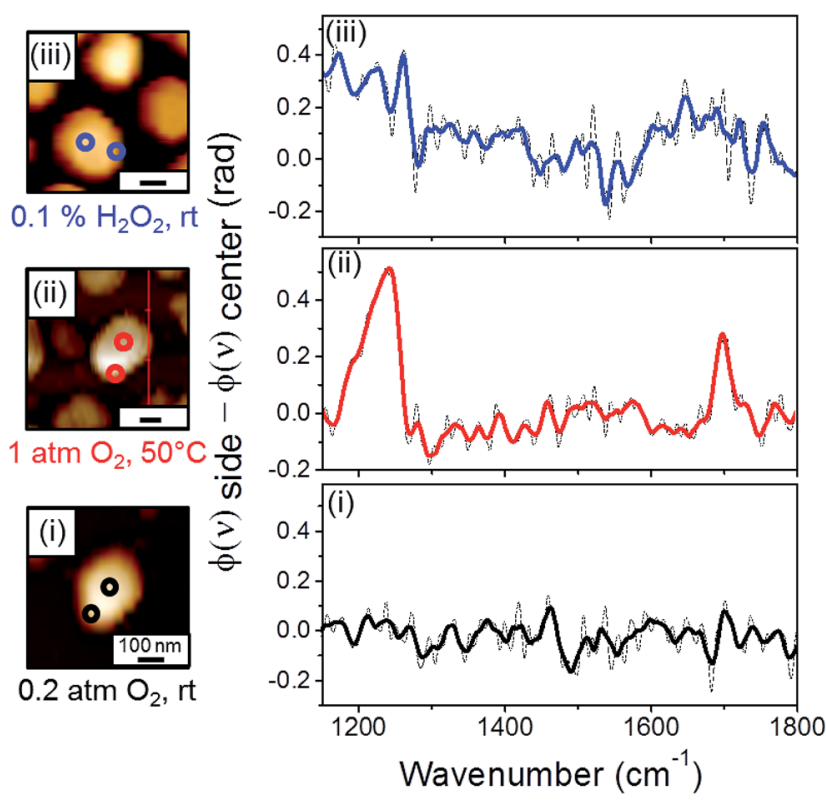

Fig. 3 Infrared nanospectroscopy point measurements were conducted on the edge and center of single NHC-coated Pt particles. The location of IR measurements is marked by colored circles in the AFM images (left panels). The differences in near-field spectra that were acquired on the side $\left(\varphi_{(\nu) \text { side }}\right)$ and center $\left(\varphi_{(\nu) \text { center }}\right)$ of each particle are reported (right panels). IR nanospectroscopy measurements were conducted following NHCs' deposition (i) and after exposure of the sample to gas phase ( $1 \mathrm{~atm}$ of $\mathrm{O}_{2}, 50{ }^{\circ} \mathrm{C}, 10 \mathrm{~h}$ ) (ii) and liquid phase $(0.1 \%$ w/w $\mathrm{H}_{2} \mathrm{O}_{2}, \mathrm{rt}, 10 \mathrm{~h}$ ) (iii) oxidizing conditions. The dash and color lines represent the as-received and smoothed spectra, respectively.

high density of low-coordinated metal atoms at the particle's edge ${ }^{40}$ Cross section and profile analysis of the supported Pt particles was performed (ESI Fig. S4 $\dagger$ ). Based on analysis of several probed particles it was identified that the height of the particles was $25 \pm 5 \mathrm{~nm}$, while their profile length was $20 \pm$ $5 \mathrm{~nm}$. Such profile length within non-crystalline particles will induce corrugated edges with high density of low-coordinated surface atoms.

As previously demonstrated, ${ }^{\mathbf{4 1}}$ a relatively small increase in the density of low-coordinated surface atoms can induce a major enhancement in the catalytic reactivity. Thus, a local increase in the density of defects on the periphery of the particle, in comparison to their density on the center of the particle, can lead to the enhanced reactivity on these surface sites, which was detected by conducting site-dependent IR nanospectroscopy measurements. In addition, it cannot be excluded that the edges of the particle expose various low index surface planes that are different and more reactive than those located on the flat center of the particle and induce the observed enhancement of the catalytic reactivity on the particle's periphery.

Site-dependent reactivity was also observed after exposure of the sample to liquid phase oxidizing conditions $(0.1 \% \mathrm{w} / \mathrm{w}$ $\mathrm{H}_{2} \mathrm{O}_{2}$, rt, $10 \mathrm{~h}$ ) (Fig. 3, spectrum (iii)). Interestingly, the obtained difference spectrum displayed a lineshape distinct from that detected after gas-phase oxidation: the $\mathrm{C}=\mathrm{O}$ contribution was broader and shifted to lower energy (1570-1720 $\mathrm{cm}^{-1}$ ), while the sharp $\mathrm{C}-\mathrm{OH}$ peak was smaller and shifted to $1250 \mathrm{~cm}^{-1}$. The chemical interpretation for the observed changes of peak energy and intensity will be discussed later.

The side-center difference spectra (Fig. 3) nicely demonstrate that exposure of the sample to gas- or liquid-phase oxidizing conditions had a different influence on the chemical properties of molecules that were anchored on the perimeter of the particle. These chemical differences were not observed while sampling NHCs that were anchored on the center of the particle (Fig. 2, spectrum (ii) and (iii), respectively).

Exposure of the gas phase oxidized sample to harsher reducing conditions ( $1 \mathrm{~atm} \mathrm{H}_{2}, 80{ }^{\circ} \mathrm{C}, 10 \mathrm{~h}$ ) diminished the chemical differences between NHCs that were anchored to the side and center of the particle (Fig. S5 $\dagger$ ). This result shows that the signals in the difference spectra (Fig. 3) were induced by variations in the chemical properties of surface-anchored NHCs and not by changes in the density or orientation of NHCs on different surface sites.

Following the results obtained by SINS measurements, complementary spatially averaged IRRAS and XPS measurements were conducted to better identify the reasons for the detected changes in the SINS spectrum at different surface sites (Fig. 4). IRRAS spectra of the as-deposited NHCs featured a dominant peak at $1250 \mathrm{~cm}^{-1}$, which was assigned to $\mathrm{C}-\mathrm{OH}$ vibration (Fig. 4a, spectrum (i)). N1s XP-spectrum of the asdeposited NHCs was measured (Fig. 4b, spectrum (i)) and its peak area was fit by two Gaussians (Fig. S6†) centered at 401.2 and $403.7 \mathrm{eV}$, which were assigned to $\mathrm{N}-\mathrm{C}$ and $\mathrm{N}-\mathrm{O}_{x} /$ amide species with a spectral weight of $86 \%$ and $14 \%$ of the total peak area, respectively (Table 1). The detection of a relatively wide $(F W H M=3 \mathrm{eV})$ N1s XPS peak of surface anchored NHCs was previously reported..$^{25,32,42} \mathrm{C} 1 \mathrm{~s}$ XP-spectrum (Fig. 4c, spectrum (i)) was fit by two Gaussians (Fig. S5†), centered at 285.5 and $286.6 \mathrm{eV}$, which correspond to $\mathrm{C}-\mathrm{C}$ and $\mathrm{C}-\mathrm{O} / \mathrm{N}$ species with a spectral weight of $44 \%$ and $56 \%$, respectively (Table 1 ).

The lack of IR peaks in the $\mathrm{C}=\mathrm{O}$ region and the absence of a $\mathrm{COOH}$ feature in the C1s XPS peak indicate that under ambient conditions most of the surface-anchored NHCs were $\mathrm{OH}$-terminated and were not oxidized. The detection of a minor $\mathrm{C}=\mathrm{O}$ vibration signal $\left(\sim 1700 \mathrm{~cm}^{-1}\right)$ in the IR nanospectroscopy measurement (Fig. 2, spectrum (i)), which was not observed in the IRRAS measurement (Fig. 4a, spectrum (i)), can be correlated to the enhanced sensitivity of IR nanospectroscopy measurements to dipoles that are positioned vertical to the surface, thus making it feasible to detect the contribution of this minor species by near-field IR nanospectroscopy.

The detection of a small fraction $(<15 \%)$ of high binding energy nitrogen species on the surface, correlated to $\mathrm{N}-\mathrm{O}_{x}$ or amide, can be induced by the strong interaction between the NHCs and the Pt surface which led to partial deformation of the surface anchored NHCs.

NEXAFS measurements at the carbon K-edge were performed to identify the geometry of surface anchored NHCs. By changing the orientation of the probed surface with respect to the linearly polarized photon beam, both p- and s-polarized NEXAFS spectra (marked in Fig. 5 with solid and dotted lines, 

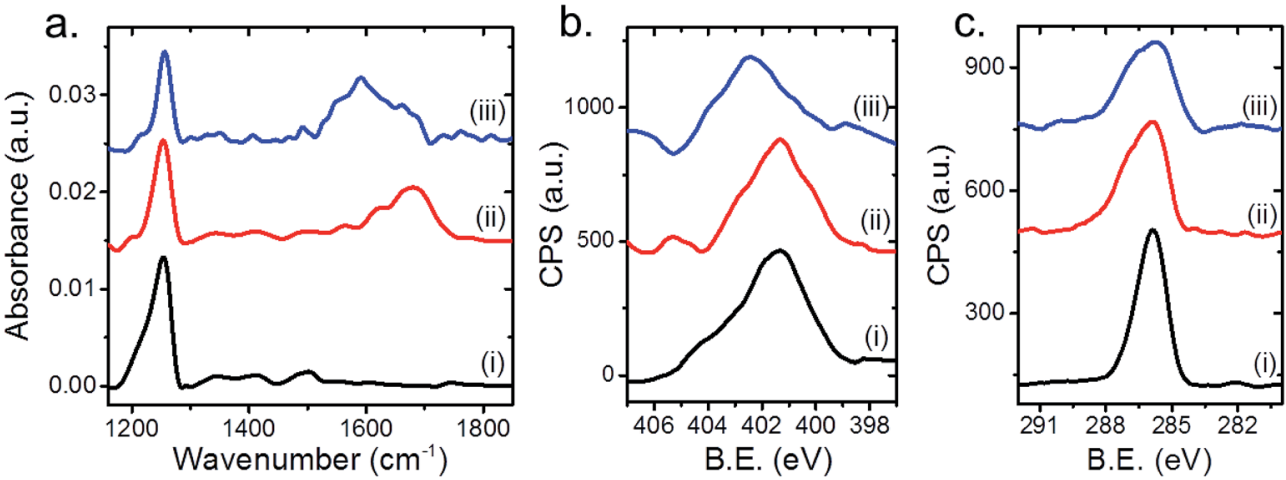

Fig. 4 IRRAS (a), N1s XPS (b) and C1s XPS (c) spectra of OH-functionalized NHCs that were anchored to the surface of supported Pt particles. Spectroscopic measurements were conducted before (i) and after exposure of the sample to gas phase $\left(1 \mathrm{~atm} \mathrm{O}_{2}, 80^{\circ} \mathrm{C}, 10 \mathrm{~h}\right)$ (ii) and liquid phase ( $0.1 \% \mathrm{w} / \mathrm{w} \mathrm{H}_{2} \mathrm{O}_{2}, \mathrm{rt}, 10 \mathrm{~h}$ ) (iii) oxidizing conditions.

respectively) were acquired. The main features in the p- and spolarized NEXAFS spectra of the as-deposited OH-NHCs (Fig. 5, spectra (i), solid and dotted lines, respectively) were associated to the $\mathrm{C} 1 \mathrm{~s} \rightarrow \sigma_{(\mathrm{C}-\mathrm{H})}^{*}(287.5 \mathrm{eV})$ and to $\sigma_{(\mathrm{C}-\mathrm{C})}^{*}$ or/and $\sigma_{(\mathrm{C}-\mathrm{O})}^{*}$ transitions (at $\left.289-293 \mathrm{eV}\right){ }^{43-45}$ In addition, a peak was detected in the s-polarized NEXAFS spectrum at $285.2 \mathrm{eV}$, which was not detected in the p-polarized spectrum and was assigned to $\pi_{(\mathrm{C}=\mathrm{H})}^{*}$ transitions. ${ }^{46,47}$ This dichroism implies that the imidazoline rings of the as-deposited NHCs display a preferential orientation perpendicular to the surface.

Exposure of the sample to gas-phase oxidizing conditions (1 atm $\mathrm{O}_{2}, 80{ }^{\circ} \mathrm{C}, 10 \mathrm{~h}$ ) induced the formation of a new IR peak at $1600-1730 \mathrm{~cm}^{-1}$ (Fig. 4a, spectrum (ii)). The detected IRRAS spectrum is complementary to the measured SINS spectrum (Fig. 3, spectrum (ii)) and indicates that exposure of the sample to gas phase oxidizing conditions induced a dominant IR absorption peak in the $\mathrm{C}=\mathrm{O}$ region.

The oxidation of the NHCs' hydroxyl groups was also identified in the C1s XP-spectrum (Fig. 4c, spectrum (ii)). The C1s XP spectrum was fit to three Gaussians, centered at 285.5 and 286.5 and $288.4 \mathrm{eV}$, corresponding to $\mathrm{C}-\mathrm{C}, \mathrm{C}-\mathrm{O} / \mathrm{N}$ and $\mathrm{COOH}$ species with spectral weight of $32 \%, 60 \%$ and $8 \%$, respectively (Fig. S6 $\dagger$ ). The detection of a higher binding energy species in the C1s XP-spectra indicated that oxidation of the $\mathrm{NHCs}^{\prime} \mathrm{OH}^{-}$ functional groups has occurred following exposure to gas phase oxidizing conditions.

NEXAFS measurements revealed that exposure of the sample to gas phase oxidizing conditions led to the development of

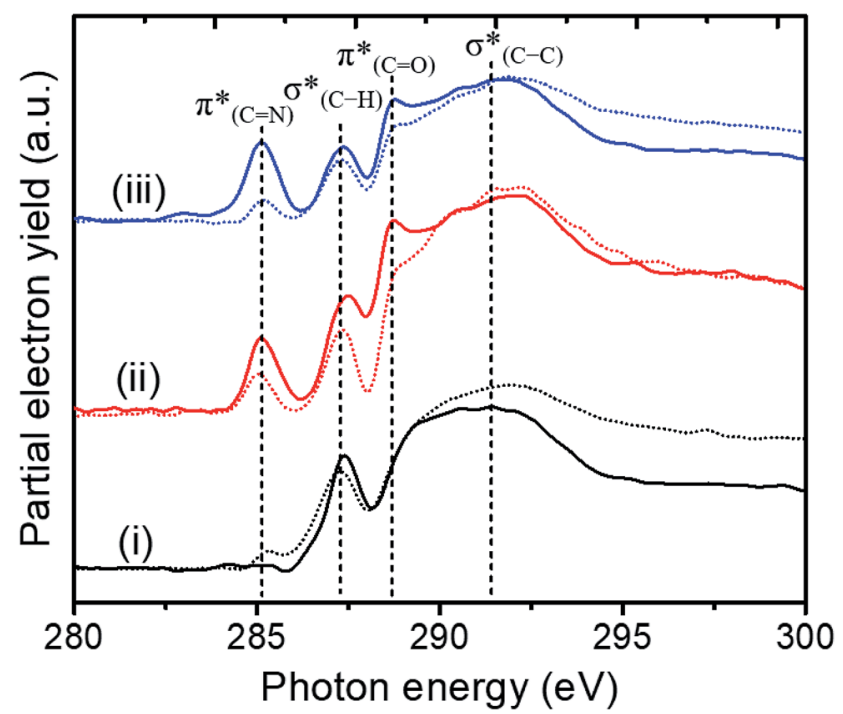

Fig. 5 Carbon $\mathrm{K}$-edge NEXAFS spectra of $\mathrm{OH}$-functionalized NHCs that were anchored to the surface of Si-supported Pt particles. $\mathrm{p}$ - and s-polarized NEXAFS spectra (marked by solid and dotted lines, respectively) were acquired by changing the orientation of the probed surface with respect to the X-ray beam. NEXAFS measurements were performed on the as-deposited NHCs (i) and after exposure of the sample to gas phase $\left(1 \mathrm{~atm} \mathrm{O}_{2}, 80{ }^{\circ} \mathrm{C}, 10 \mathrm{~h}\right)$ (ii) and liquid phase $(0.1 \%$ w/w $\mathrm{H}_{2} \mathrm{O}_{2}, \mathrm{rt}, 10 \mathrm{~h}$ ) (iii) oxidizing conditions.

a new peak in the p-polarized spectrum at $288.8 \mathrm{eV}$ (Fig. 5, spectra (ii), solid line), which was associated to a $\pi_{(\mathrm{C}=\mathrm{O})}^{*}$ transition. ${ }^{48}$ The appearance of the $\pi_{(\mathrm{C}=\mathrm{O})}^{*}$ resonance and the

Table 1 Analysis of the C1s and N1s XPS peak area

XPS signal

\begin{tabular}{|c|c|c|c|c|c|c|}
\hline \multirow[b]{2}{*}{ Treatment } & \multicolumn{3}{|l|}{ N1s } & \multicolumn{3}{|l|}{ C1s } \\
\hline & $\begin{array}{l}\mathrm{N}=\mathrm{C} \\
(398.5 \mathrm{eV})\end{array}$ & $\begin{array}{l}\mathrm{N}-\mathrm{C} \\
(401.2 \mathrm{eV})\end{array}$ & $\begin{array}{l}\text { Amide } / \mathrm{N}-\mathrm{O}_{x} \\
(402.7-403.7 \mathrm{eV})\end{array}$ & $\begin{array}{l}\mathrm{C}-\mathrm{C} \\
(285.5 \mathrm{eV})\end{array}$ & $\begin{array}{l}\mathrm{C}-\mathrm{O} / \mathrm{N} \\
(286.6 \mathrm{eV})\end{array}$ & $\begin{array}{l}\mathrm{COOH} \\
(288.4 \mathrm{eV})\end{array}$ \\
\hline Air, rt & - & $86 \%$ & $14 \%$ & $44 \%$ & $56 \%$ & - \\
\hline Gas phase oxidation & $5 \%$ & $83 \%$ & $12 \%$ & $32 \%$ & $60 \%$ & $8 \%$ \\
\hline Liquid phase oxidation & $10 \%$ & $30 \%$ & $60 \%$ & $33 \%$ & $60 \%$ & $7 \%$ \\
\hline
\end{tabular}


decrease of the $\sigma_{(\mathrm{C}-\mathrm{H})}^{*}$ one in the NEXAFS spectra indicates that the NHCs' hydroxyl functional groups were oxidized following exposure of the sample to gas-phase oxidizing conditions. Thus, the analysis of the X-ray spectroscopy shows that exposure of the sample to gas phase oxidizing conditions induced oxidation of the NHCs' hydroxyl functional groups into a carboxylic acid. These results nicely correlate to SINS measurements, which identified enhanced hydroxyl oxidation rate on the periphery of the particles (Fig. 3, spectrum (ii)).

The N1s XP-spectrum (Fig. 4b, spectrum (ii)) included a tail toward lower binding energy, which was not observed in the asdeposited NHCs (Fig. 4b, spectrum (i)). The latter feature was fitted by an additional Gaussian component centered at $398.5 \mathrm{eV}$ (weight of $5 \%$ ) and was associated to $\mathrm{C}=\mathrm{N}$ species. In addition, the center of the high binding energy component in the N1s XP-spectrum shifted to lower energy $(402.7 \mathrm{eV})$, in comparison to the location of this component in the asdeposited sample (Fig. S6 $\dagger$ ).

Exposure of the sample to oxidizing conditions also led to a noticeable increase in the intensity of the $\pi_{(\mathrm{C}=\mathrm{N})}^{*}$ resonance at $285.2 \mathrm{eV}$. Transitions in this region are usually attributed to aromatic rings. ${ }^{49}$ We therefore conclude that exposure of the sample to oxidizing conditions facilitated oxidative dehydrogenation reaction of the imidazoline ring, resulting in increased unsaturation and aromaticity of the molecule. ${ }^{50}$ The increased aromaticity of the sample can potentially induce the observed shift in the position of the high binding energy fraction of the N1s spectra to lower energy values.

Comparison of the p- and s-polarized NEXAFS spectra (Fig. 5, spectra (ii), solid and dotted lines, respectively) reveals that both $\pi_{(\mathrm{C}=\mathrm{N})}^{*}$ and $\pi_{(\mathrm{C}=\mathrm{O})}^{*}$ resonances (285.2 and $288.8 \mathrm{eV}$, respectively) are more intense in the p-polarized NEXAFS spectrum. This dichroism implies that after exposure of the sample to gasphase oxidizing conditions, NHCs display a preferential orientation parallel to the Pt surface. ${ }^{51}$

It should be noted that spatially averaged IRRAS, XPS and NEXAFS measurements were performed after exposure of the sample to gas-phase oxidizing conditions $\left(1 \mathrm{~atm} \mathrm{O}_{2}, 80^{\circ} \mathrm{C}, 10 \mathrm{~h}\right)$ which were harsher than those adopted prior to SINS measurement $\left(1 \mathrm{~atm} \mathrm{O}_{2}, 50^{\circ} \mathrm{C}, 10 \mathrm{~h}\right)$. Exposure of the sample to harsher oxidizing conditions ensured that most of the surface anchored NHCs were oxidized into acid. ${ }^{23}$ Thus, the spatially averaged spectroscopic data can be directly compared to SINS measurements performed at the nanoparticle's periphery under milder oxidizing conditions.

Exposure of the sample to liquid phase oxidizing conditions induced a broadening of the IRRAS peak at high frequency $\left(1520-1700 \mathrm{~cm}^{-1}\right)$ and a shift of the N1s XPS peak to higher binding energy (spectrum (iii) in Fig. $4 \mathrm{a}$ and b, respectively). The N1s XPS peak was fit to three Gaussians, centered at 398.5, 401.2 and $402.7 \mathrm{eV}$, which were associated to $\mathrm{N}=\mathrm{C}, \mathrm{N}-\mathrm{C}$ and $\mathrm{N}-\mathrm{O}_{x} /$ amide species with a weight of $10 \%, 30 \%$ and $60 \%$, respectively. The corresponding C1s XPS peak was fit to three Gaussians, centered at $285.5,286.6$ and $288.4 \mathrm{eV}$, which were associated to $\mathrm{C}-\mathrm{C}, \mathrm{C}-\mathrm{O} / \mathrm{N}$ and $\mathrm{COOH}$ species with a weight of $33 \%, 60 \%$ and $7 \%$, respectively (Fig. 4c, curve (iii)). The C1s XP-spectrum from the sample exposed to liquid phase oxidizing conditions provides results qualitatively similar to those obtained after exposure of the sample to gas phase oxidizing conditions, however the total area of the former peak decreased by $\sim 15 \%$ in comparison to the one measured after gas phase reaction conditions.

The shift to higher energy observed in the N1s XPS peak and that to lower energy of the high frequency IR peak of the liquid phase oxidized sample is consistent with decarboxylation followed by oxidation of the NHCs. The decarboxylation results in the observed decrease in the C1s XPS peak area (spectrum (iii) in Fig. 4c) and the overall decrease of the $\sigma_{(\mathrm{C}-\mathrm{H})}^{*}(287.5 \mathrm{eV})$ and $\sigma_{(\mathrm{C}-\mathrm{C})}^{*}($ at $289-293 \mathrm{eV})$ resonances in the NEXAFS spectrum (Fig. 5, spectrum (iii)).

Analysis of the N1s to Pt4f peaks area ratio (Fig. S7 $\dagger$ ) revealed that exposure of the sample to either gas or liquid phase oxidizing conditions induced relatively small changes $(<10 \%)$ in the N1s/Pt4f peaks ratio. This analysis verifies that exposure of the sample to different oxidizing conditions did not lead to a noticeable detachment of the NHCs' imidazoline ring from the Pt surface, in agreement with a previous report. ${ }^{32}$

Exposure of the sample to liquid phase oxidizing conditions led to an overall increase of the $\pi_{(\mathrm{C}=\mathrm{N})}^{*}$ resonance. This result can be attributed to an increase in the aromatization of the imidazole ring resulting from oxidative dehydrogenation of NHC imidazoline. This hypothesis is supported by the detection of a higher fraction of $\mathrm{C}=\mathrm{N}$ species in the N1s XPS peak (Table 1)..$^{52-54}$ The dichroism of the NEXAFS spectra, in particular of the $\pi_{(\mathrm{C}=\mathrm{N})}^{*}$ and $\pi_{(\mathrm{C}=\mathrm{O})}^{*}$ resonances, was increased upon exposure of the sample to liquid phase oxidizing conditions (Fig. 5, spectrum (iii), solid and dotted lines, respectively). This large dichroism indicates that exposure of the sample to liquid phase oxidizing conditions favored a tilting of the oxidized NHCs' imidazole rings to an orientation preferentially parallel to the Pt surface.

From the analysis of the spectroscopic data, we conclude that exposure of the sample to liquid phase oxidizing conditions induced two main chemical changes: (1) decarboxylation and oxidation of the NHCs' functional group, which preferentially occurs on the nanoparticles' periphery. (2) Enhanced oxidative dehydrogenation reaction that increased the aromaticity of the molecule. These two effects were coupled to a change of the adsorption geometry corresponding to a change of the preferential orientation of the oxidized NHCs' imidazole ring and of the carbonyl group from perpendicular to parallel to the Pt surface.

\section{Conclusions}

In this work surface anchored NHCs as chemical indicators were employed to interrogate the site-dependent reactivity of oxidation reactions on single Pt particles. Thus, the integration of spatially localized and averaged measurements provided a detailed analysis of the influence of reaction conditions on the site-dependent reactivity and on the geometry of NHCs on Pt particles. More specifically, integration of high spatial resolution SINS measurements and spatially averaged XPS, NEXAFS and IRRAS measurements provide evidence that, under gas 
phase oxidizing conditions, the hydroxyl groups of surfaceanchored NHCs at the periphery of Pt particles showed enhanced reactivity relative to those located at the center, thus leading to the formation of carboxylic acid substituted NHCs. Importantly, this enhancement in reactivity was detected at NHCs located at the periphery of the particles; however, this differential reactivity was highly dependent on the reaction conditions. On the other hand, no differences were observed in the chemical properties of NHCs that were anchored on the center of the Pt particles and were exposed to either gas or liquid phase oxidizing conditions. Exposure of the sample to harsher, liquid phase oxidizing conditions, using $\mathrm{H}_{2} \mathrm{O}_{2}$ as oxidant, resulted in modification of the NHCs located at the periphery of the nanoparticles. Moreover, exposure of the sample to either gas or liquid phase oxidizing conditions induced oxidative dehydrogenation reaction of the imidazoline ring that led to a favorable preferential orientation of the now oxidized NHC imidazole rings parallel to the surface. The dehydrogenation reaction was not observed in the hydroxyl functionalized NHCs prior to their exposure to oxidizing conditions. While NHC-substituted surfaces are gaining prominence in catalysis, this observation highlights that in some cases, these ligands may be reactive under reaction condition. Finally, this work highlights that the observation of differential reactivity at sites in heterogeneous catalysts should be examined and expressed in, and maybe limited to, the context of given reaction conditions.

\section{Conflicts of interest}

There are no conflicts to declare.

\section{Acknowledgements}

This research was supported by Grant No. 2016344 from the United States-Israel Binational Science Foundation (BSF). S. D. acknowledges the Israeli Ministry of Energy and the Rudin fellowship for their financial support. F. D. T. thanks the Director, Office of Science, Office of Basic Energy Sciences and the Division of Chemical Sciences, Geosciences, and Biosciences of the US Department of Energy at LBNL (DE-AC0205CH11231) for partial support of this work. The Advanced Light Source is supported by the Director, Office of Science, Office of Basic Energy Sciences, of the US Department of Energy under contract number DE-AC02-05CH11231. We acknowledge the assistance of Dr Hans Bechtel (Advanced Light Source, Lawrence Berkeley National Lab) in conducting the SINS experiments.

\section{References}

1 G. A. Somorjai and Y. Li, Introduction to surface chemistry and catalysis, John Wiley \& Sons, 2010.

2 H. J. Freund, Chemistry, 2010, 16, 9384-9397.

3 G. Ertl, Angew. Chem., Int. Ed., 2008, 47, 3524-3535.
4 I. Chorkendorff and J. W. Niemantsverdriet, Concepts of modern catalysis and kinetics, Wiley-VCH, Weinheim, 2nd edn, 2007.

5 K. F. Kalz, R. Kraehnert, M. Dvoyashkin, R. Dittmeyer, R. Glaser, U. Krewer, K. Reuter and J. D. Grunwaldt, ChemCatChem, 2017, 9, 17-29.

6 L. D. Pachón and G. Rothenberg, Appl. Organomet. Chem., 2008, 22, 288-299.

7 G. A. Somorjai and J. Y. Park, Angew. Chem., Int. Ed., 2008, 47, 9212-9228.

8 I. L. C. Buurmans and B. M. Weckhuysen, Nat. Chem., 2012, 4, 873-886.

9 W. Wang and N. J. Tao, Anal. Chem., 2014, 86, 2-14.

10 W. L. Xu, J. S. Kong, Y. T. E. Yeh and P. Chen, Nat. Mater., 2008, 7, 992-996.

11 M. A. Bañares, Catal. Today, 2005, 100, 71-77.

12 E. Stavitski and B. M. Weckhuysen, Chem. Soc. Rev., 2010, 39, 4615-4625.

13 E. Gross, X. Z. Shu, S. Alayoglu, H. A. Bechtel, M. C. Martin, F. D. Toste and G. A. Somorjai, J. Am. Chem. Soc., 2014, 136, 3624-3629.

14 Y. Levartovsky and E. Gross, Top. Catal., 2016, 59, 1700-1711. 15 E. Gross, Surf. Sci., 2016, 648, 136-140.

16 R. W. Johns, H. A. Bechtel, E. L. Runnerstrom, A. Agrawal, S. D. Lounis and D. J. Milliron, Nat. Commun., 2016, 7, 11583.

17 F. Huth, A. Govyadinov, S. Amarie, W. Nuansing, F. Keilmann and R. Hillenbrand, Nano Lett., 2012, 12, 3973-3978.

18 N. Kumar, B. Stephanidis, R. Zenobi, A. J. Wain and D. Roy, Nanoscale, 2015, 7, 7133-7137.

19 H. A. Bechtel, E. A. Muller, R. L. Olmon, M. C. Martin and M. B. Raschke, Proc. Natl. Acad. Sci. U. S. A., 2014, 111, 7191-7196.

20 A. B. Zrimsek, N. H. Chiang, M. Mattei, S. Zaleski, M. O. McAnally, C. T. Chapman, A. I. Henry, G. C. Schatz and R. P. Van Duyne, Chem. Rev., 2017, 117, 7583-7613.

21 E. M. van Schrojenstein Lantman, T. Deckert-Gaudig, A. J. G. Mank, V. Deckert and B. M. Weckhuysen, Nat. Nanotechnol., 2012, 7, 583-586.

22 J. H. Zhong, X. Jin, L. Y. Meng, X. Wang, H. S. Su, Z. L. Yang, C. T. Williams and B. Ren, Nat. Nanotechnol., 2017, 12, 132136.

23 C. Y. Wu, W. J. Wolf, Y. Levartovsky, H. A. Bechtel, M. C. Martin, F. D. Toste and E. Gross, Nature, 2017, 541, 511-515.

24 D. L. Fu, K. Park, G. Delen, O. Attila, F. Meirer, D. Nowak, S. Park, J. E. Schmidt and B. M. Weckhuysen, Chem. Commun., 2017, 53, 13012-13014.

25 Y. Levratovsky and E. Gross, Faraday Discuss., 2016, 188, 345-353.

26 W. Wang, Chem. Soc. Rev., 2018, 47, 2485-2508.

27 H. Topsøe, J. Catal., 2003, 216, 155-164.

28 H.-F. Wang and Z.-P. Liu, J. Am. Chem. Soc., 2008, 130, 10996-11004.

29 H. Wang, A. Sapi, C. M. Thompson, F. Liu, D. Zherebetskyy, J. M. Krier, L. M. Carl, X. Cai, L.-W. Wang and G. A. Somorjai, J. Am. Chem. Soc., 2014, 136, 10515-10520. 
30 Z. Ristanovic, A. V. Kubarev, J. Hofkens, M. B. J. Roeffaers and B. M. Weckhuysen, J. Am. Chem. Soc., 2016, 138, 13586-13596.

31 M. N. Hopkinson, C. Richter, M. Schedler and F. Glorius, Nature, 2014, 510, 485-496.

32 C. M. Crudden, J. H. Horton, I. I. Ebralidze, O. V. Zenkina, A. B. McLean, B. Drevniok, Z. She, H. B. Kraatz, N. J. Mosey, T. Seki, E. C. Keske, J. D. Leake, A. RousinaWebb and G. Wu, Nat. Chem., 2014, 6, 409-414.

33 A. V. Zhukhovitskiy, M. G. Mavros, T. Van Voorhis and J. A. Johnson, J. Am. Chem. Soc., 2013, 135, 7418-7421.

34 A. Ruhling, K. Schaepe, L. Rakers, B. Vonhoren, P. Tegeder, B. J. Ravoo and F. Glorius, Angew. Chem., Int. Ed., 2016, 55, 5856-5860.

35 J. B. Ernst, C. Schwermann, G. I. Yokota, M. Tada, S. Muratsugu, N. L. Doltsinis and F. Glorius, J. Am. Chem. Soc., 2017, 139, 9144-9147.

36 C. R. Larrea, C. J. Baddeley, M. R. Narouz, N. J. Mosey, J. H. Horton and C. M. Crudden, ChemPhysChem, 2017, 18, 3536-3539.

37 G. Q. Wang, A. Ruhling, S. Amirjalayer, M. Knor, J. B. Ernst, C. Richter, H. J. Gao, A. Timmer, H. Y. Gao, N. L. Doltsinis, F. Glorius and H. Fuchs, Nat. Chem., 2017, 9, 152-156.

38 L. Jiang, B. D. Zhang, G. Medard, A. P. Seitsonen, F. Haag, F. Allegretti, J. Reichert, B. Kuster, J. V. Barth and A. C. Papageorgiou, Chem. Sci., 2017, 8, 8301-8308.

39 L. Floreano, A. Cossaro, R. Gotter, A. Verdini, G. Bavdek, F. Evangelista, A. Ruocco, A. Morgante and D. Cvetko, J. Phys. Chem. C, 2008, 112, 10794-10802.
40 N. Lopez, T. V. W. Janssens, B. S. Clausen, Y. Xu, M. Mavrikakis, T. Bligaard and J. K. Norskov, J. Catal., 2004, 223, 232-235.

41 G. A. Somorjai and D. W. Blakely, Nature, 1975, 258, 580-583. 42 J. B. Ernst, S. Muratsugu, F. Wang, M. Tada and F. Glorius, J. Am. Chem. Soc., 2016, 138, 10718-10721.

43 Y. H. La, Y. J. Jung, T. H. Kang, K. Ihm, K. J. Kim, B. Kim and J. W. Park, Langmuir, 2003, 19, 9984-9987.

44 D. A. Outka, J. Stohr, J. P. Rabe and J. D. Swalen, J. Chem. Phys., 1988, 88, 4076-4087.

45 M. Beccari, A. Kanjilal, M. G. Betti, C. Mariani, L. Floreano, A. Cossaro and V. Di Castro, J. Electron Spectrosc. Relat. Phenom., 2009, 172, 64-68.

46 A. L. Johnson, E. L. Muetterties, J. Stohr and F. Sette, J. Phys. Chem., 1985, 89, 4071-4075.

47 N. Graf, E. Yegen, T. Gross, A. Lippitz, W. Weigel, S. Krakert, A. Terfort and W. E. S. Unger, Surf. Sci., 2009, 603, 28492860.

48 A. Cossaro, S. Terreni, O. Cavalleri, M. Prato, D. Cvetko, A. Morgante, L. Floreano and M. Canepa, Langmuir, 2006, 22, 11193-11198.

49 K. G. Latham, M. I. Simone, W. M. Dose, J. A. Allen and S. W. Donne, Carbon, 2017, 114, 566-578.

50 D. W. Blakely and G. A. Somorjai, J. Catal., 1976, 42, 181-196. 51 U. Siemeling, H. Memczak, C. Bruhn, F. Vogel, F. Trager, J. E. Baio and T. Weidner, Dalton Trans., 2012, 41, 29862994.

52 R. J. J. Jansen and H. Vanbekkum, Carbon, 1995, 33, 10211027.

53 R. V. Duevel and R. M. Corn, Anal. Chem., 1992, 64, 337-342. 54 B. L. Frey and R. M. Corn, Anal. Chem., 1996, 68, 3187-3193. 